EDITORIAL

\title{
Awaiting the perfect diagnostic test: optimal prostate cancer care begins without a diagnosis
}

(c) The Author(s), under exclusive licence to Springer Nature Limited 2022

Prostate Cancer and Prostatic Diseases (2022) 25:135-136; https:// doi.org/10.1038/s41391-022-00503-y

Given the limitations of PSA as a marker for prostate cancer, there is a critical need for diagnostic tools that can reduce the use of unnecessary biopsies while preserving early detection of potentially-lethal cancers. In patients presenting with elevated PSA, the current diagnostic approach suggests that clinicians offer multi-parametric magnetic resonance imaging (mpMRI) and consider serum- or urine-based biomarkers to better define the risk of high-grade cancer prior to biopsy [1]. Still, the optimal use and interpretation of current diagnostic tools are not clearly defined, in large part due to the limitations of available clinical data [2].

In the current report, Hendriks et al. performed both urinebased SelectMDx-testing and mpMRI prior to biopsy in 599 biopsy-naïve men with $P S A \geq 3 \mathrm{ng} / \mathrm{ml}$ [3]. All men underwent systematic biopsy, and patients with suspicious mpMRI also underwent MR-guided biopsy. The authors then assessed projected clinical outcomes under each of four potential testing strategies: (1) SelectMDx-testing-only, with biopsy performed if the SelectMDx test was positive; (2) mpMRI-only, with biopsy performed if mpMRI was positive (PI-RADS $\geq 3$ ); (3) SelectMDxtesting followed by mpMRI if the SelectMDx test was positive, and biopsy performed if mpMRI was positive (conditionalstrategy; biopsy performed only if both tests were positive), and (4) SelectMDx-testing and mpMRI in all patients, with biopsy performed if either test was positive (joint-strategy). Projected outcomes included the number of biopsies avoided, detection of high-grade (Grade Group [GG] $\geq 2$ ) cancer, and detection of lowgrade (GG1) cancer. SelectMDx was considered negative for scores less than -2.8 , a cutoff associated with a $13 \%$ risk of detecting $G G \geq 2$ cancer on biopsy after a negative test [4].

Using each strategy, the proportion of men who would have undergone SelectMDx testing and mpMRI are listed with the subsequent clinical outcomes in the Table 1 As could be expected, the sensitivity for detecting GG $\geq 2$ cancer was associated with the proportion of patients who underwent biopsy under each strategy. For example, the joint strategy - under which patients proceeded to biopsy if either SelectMDx or mpMRI were positive led to the highest rate of biopsy (72\%) and the highest rate of detecting GG $\geq 2$ cancer (98\%). The conditional strategy - under which biopsy was performed only if both SelectMDx and mpMRI were positive-led to the lowest rate of biopsy (40\%) and the lowest rate of $\mathrm{GG} \geq 2$ cancer detection (87\%), while notably also providing the greatest reduction in overdiagnosis of GG1 cancers (58\% reduction). On decision curve analysis, the mpMRI-only strategy-under which $51 \%$ of men underwent biopsy and $95 \%$ of GG $\geq 2$ cancers were detected-demonstrated the highest net benefit. The conditional strategy (i.e. biopsy performed only if both tests were positive) provided the second-highest net benefit across the majority of risk thresholds.

The use of mpMRI has been shown to improve diagnostic yield of prostate biopsy and has emerged as a key component of diagnostic testing [5]. Clinically, the greatest concern with population-wide adoption of mpMRI is its highly variable accuracy across institutions and among individual radiologists [6, 7]. For example, Sonn et al. found the negative predictive value (NPV) of mpMRI for GG $\geq 2$ cancer ranged from 40 to $87 \%$ across radiologists at a single academic center [6]. In a recent meta-analysis, Sathianathen et al observed a pooled NPV approximating $90 \%$, although published values ranged as low as $62 \%$ by study [7]. Moreover, these data were obtained largely from experienced academic centers, as the authors acknowledged there are insufficient published data for planned analyses of nonacademic centers. Notably, the positive predictive value (PPV) of mpMRI appears to be similarly variable. In a meta-analysis of 26 experienced imaging centers [8], PI-RADS 4 lesions had an estimated overall PPV of $39 \%$, with an interquartile range (IQR) of PPVs extending from 25 to $55 \%$. Similarly, the interquartile range of PPVs for PI-RADS 5 lesions extended from 61 to $82 \%$ across centers. As previous authors have emphasized [7], in light of inter- and intra-institutional variability of $\mathrm{mpMRI}$ reading and interpretation, determining the potential utility of mpMRI in a given practice setting requires knowledge of local mpMRI data.

Considering these data and practical limitations of mpMRI (e.g., access to high-quality imaging; adherence to technical standards; time, labor, and cost associated with testing) [9], objectivelymeasured biomarkers obtainable in routine practice could be more practical for initial reflex testing after PSA. In the current study, it is notable that mpMRI provided $95 \%$ sensitivity for GG $\geq 2$ disease-a value likely exceeding what would be expected from population-wide use of mpMRI $[6,7,10]$. Still, the conditional strategy of initial biomarker testing, followed by mpMRI for positive biomarker tests only, resulted in the greatest reduction in biopsy ( $60 \%$ of patients avoided biopsy), the greatest reduction in overdiagnosis of GG1 cancers (58\%), and maintained reasonably high detection of $G G \geq 2$ cancers (87\%). As the authors report, this approach provided the second-highest net benefit across the majority of pertinent risk thresholds. Thus, a conditional, biomarker-first testing approach - likely to be most feasible for population-level application-may also prove most clinicallybeneficial under "real-world" (i.e. de-centralized) interpretation of MRI or using an alternative biomarker (or alternative cutoff of the current biomarker) with different performance metrics [11].

Ultimately, the authors are to be commended for this wellperformed, prospective assessment of two clinically-available diagnostic tools. While additional studies, including head-tohead comparisons and cost-effectiveness analyses, will continue to inform the optimal diagnostic approach, these prospective data provide benchmarks of relative risks and benefits under combined testing approaches. As clinicians, a working knowledge of such 
Table 1. Tests performed and projected clinical outcomes under four diagnostic testing strategies.

\begin{tabular}{|c|c|c|c|c|c|}
\hline \multirow[b]{2}{*}{ Testing strategy } & \multicolumn{2}{|c|}{ Determined by testing strategy } & \multicolumn{3}{|c|}{ Clinical impact of test results } \\
\hline & $\begin{array}{l}\text { SelectMDx } \\
\text { performed (\%) }\end{array}$ & $\begin{array}{l}\text { mpMRI } \\
\text { performed (\%) }\end{array}$ & $\begin{array}{l}\text { Biopsies } \\
\text { avoided (\%) }\end{array}$ & $\begin{array}{l}\text { GG } \geq 2 \text { PCa detected } \\
\text { (i.e., sensitivity) }\end{array}$ & $\begin{array}{l}\text { Reduction in } \\
\text { overdiagnosis of } \\
\text { GG1 PCa }\end{array}$ \\
\hline (1) SelectMDx-only & $100 \%$ & $0 \%$ & $38 \%$ & $90 \%$ & $35 \%$ \\
\hline (2) mpMRI-only & $0 \%$ & $100 \%$ & $49 \%$ & $95 \%$ & $44 \%$ \\
\hline $\begin{array}{l}\text { (3) Conditional (biopsy if } \\
\text { both tests positive) }\end{array}$ & $100 \%$ & $62 \%$ & $60 \%$ & $87 \%$ & $58 \%$ \\
\hline $\begin{array}{l}\text { (4) Joint (biopsy if either } \\
\text { test positive) }\end{array}$ & $100 \%$ & $100 \%$ & $28 \%$ & $98 \%$ & $21 \%$ \\
\hline
\end{tabular}

data allows us to best identify which available tools can inform decision-making with the level of certainty sought by each of our patients. As the late, great Donald S. Coffey often responded when asked how much risk of prostate cancer death (in exchange for reduced morbidity) was too much risk: "That's not a medical question, that's a personal question." Until the perfect diagnostic test emerges, guiding our patients through personalized, shared decision-making will remain a most essential component of prostate cancer care.

Jeffrey J. Tosoian (iD ${ }^{1,2 凶}$

${ }^{1}$ Department of Urology, Vanderbilt University Medical Center, Nashville, TN, USA. ${ }^{2}$ Vanderbilt-Ingram Cancer Center, Nashville, TN, USA. ${ }^{\bowtie}$ email: jeff.tosoian@vumc.org

\section{REFERENCES}

1. Carroll P, Parsons J, Box G, Carlsson S, Catalona W, Dahl D, et al. NCCN clinical practice guidelines in oncology: prostate cancer early detection. 2021; 2.2021.

2. Narayan VM. A critical appraisal of biomarkers in prostate cancer. World J Urol. 2020;38:547-54.

3. Hendriks R, van der Leest MMG, Israël B, Hannink G, Setiasti AY, Cornel E, et al. Clinical use of the SelectMDx urinary biomarker test with or without mpMRI in prostate cancer diagnosis: a prospective, multicenter study in biopsy-naïve men. Prostate Cancer Prostatic Dis. 2021;24:1110-9.

4. Haese A, Trooskens G, Steyaert S, Hessels D, Brawer M, Vlaeminck-Guillem V, et al. Multicenter optimization and validation of a 2-gene mRNA urine test for detection of clinically significant prostate cancer before initial prostate biopsy. J Urol. 2019;202:256-63.

5. Ahdoot M, Wilbur AR, Reese SE, Lebastchi AH, Mehralivand S, Gomella PT, et al. MRI-targeted, systematic, and combined biopsy for prostate cancer diagnosis. N Engl J Med. 2020;382:917-28.

6. Sonn GA, Fan RE, Ghanouni P, Wang NN, Brooks JD, Loening AM, et al. Prostate magnetic resonance imaging interpretation varies substantially across radiologists. Eur Urol Focus. 2019;5:592-9.

7. Sathianathen NJ, Omer A, Harriss E, Davies L, Kasivisvanathan V, Punwani S, et al. Negative predictive value of multiparametric magnetic resonance imaging in the detection of clinically significant prostate cancer in the prostate imaging reporting and data system era: a systematic review and meta-analysis negative predictive value of Mu. Eur Urol. 2020. https://doi.org/10.1016/j.eururo.2020.03.048.

8. Westphalen AC, McCulloch CE, Anaokar JM, Arora S, Barashi NS, Barentsz JO, et al. Variability of the positive predictive value of PI-RADS for prostate mri across 26 centers: experience of the society of abdominal radiology prostate cancer disease-focused panel. Radiology. 2020;296:76-84.

9. Cuocolo R, Stanzione A, Ponsiglione A, Verde F, Ventimiglia A, Romeo V, et al. Prostate MRI technical parameters standardization: a systematic review on adherence to PI-RADSv2 acquisition protocol. Eur J Radiol. 2019;120. https://doi. org/10.1016/j.ejrad.2019.108662.

10. Leake JL, Hardman R, Ojili V, Thompson I, Shanbhogue A, Hernandez J, et al. Prostate MRI: access to and current practice of prostate MRI in the United States. J Am Coll Radio. 2014;11:156-60.

11. Eyrich NW, Morgan TM, Tosoian JJ. Biomarkers for detection of clinically significant prostate cancer: contemporary clinical data and future directions. Transl Androl Urol. 2021;10:3091-103.

\section{AUTHOR CONTRIBUTIONS}

JJT drafted this comment in reference to a recently published study (Hendricks et al, reference [3] in comment)

\section{COMPETING INTERESTS}

$J J T$ is a co-founder of LynxDx, Inc.

\section{ADDITIONAL INFORMATION}

Correspondence and requests for materials should be addressed to Jeffrey J. Tosoian.

Reprints and permission information is available at http://www.nature.com/reprints

Publisher's note Springer Nature remains neutral with regard to jurisdictional claims in published maps and institutional affiliations. 